\title{
A Hardware Efficient 64-QAM Low-IF Transceiver Baseband for Broadband Communications
}

\author{
Ching-Chi Chang, Muh-Tian Shiue*, and Chorng-Kuang Wang \\ Graduate Institute of Electronics Engineering, and Department of Electrical Engineering, \\ National Taiwan University, 106 Taipei, Taiwan R.O.C. \\ *Department of Electrical Engineering, National Central University, 320 Jung-Li, Taiwan R.O.C. \\ E-mail: ckwang@cc.ee.ntu.edu.tw
}

\begin{abstract}
This paper presents a hardware efficient VLSI design of digital baseband for 64-QAM communication systems over the lastmile cable network. This VLSI system design involves a cost-efficient architecture of the adaptive equalizer and a two-phase linear architecture of the pulse shaping filters, which reduce the hardware requirement by a factor of four comparing with traditional quadrature direct form FIR filters. In this design, the two-fold carrier recovery loop possesses a pull-in range of $\pm 100 \mathrm{kHz}$ (i.e. $\pm 18,500 \mathrm{ppm}$ of the symbol rate) and $-82 d B c$ jitter suppression. Based on the proposed multi-staged LMS-based fractionally-spaced equalizer, the receiver realizes the symbol spaced timing recovery in a $\pm 200 \mathrm{ppm}$ tolerance of the symbol rate. The acquisition time of the proposed 64-QAM blind adaptive system is $7 \mathrm{~ms}$, and the transceiver reaches the operation speed of the case for $32.28 \mathrm{Mb} / \mathrm{s}$ 64-QAM low-IF digital CATV system over NTSC $6 \mathrm{MHz}$ bandwidth channels. Using $0.35 \mu \mathrm{m}$ CMOS technology, the transceiver design occupies a chip area $5.5 \mathrm{~mm} \times 5.5 \mathrm{~mm}$ and power consumption $1.35 \mathrm{~W}(1.0 \mathrm{~W}$ for $\mathbf{R X})$ when the power supply is $3.3 \mathrm{~V}$.
\end{abstract}

\section{INTRODUCTION}

Fig.1 shows a typical functional block diagram of QAM transceiver for last-mile broadband digital communication systems, such as digital CATV [1], SCM-VDSL [2] and Wireless MAN [3]. The transmitter mainly consists of an FEC encoder, a symbol mapper, a Nyquist pulse shaping filter (PSF), a quadrature mixer, and the analog front end (AFE). In the receiver, a digital quadrature mixer and two identical low-pass filters (LPFs) are used to demodulate the received passband signal. The coherent carrier recovery (CR) loop is employed to combat the performance degradation from non-coherent receiving. The timing recovery (TR) loop for symbol timing synchronization provides the ADC a proper sampling instant. In order to combat the ISI caused by the band-limited channel, an adaptive equalizer with blind adaptation is utilized.

In a conventional architecture of a baseband adaptive QAM decision feedback equalizer (DFE), both feed forward (FF) and feedback (FB) filters contain tens to hundreds adaptive complex taps. This requires a significant hardware on an SoC chip. In order to reduce the chip area, the equalizer can employ a multiplicationand-accumulation (MAC) unit with higher clocking rate by paying the power consumption and circuit complexity [4]. Practically, this advantage will be eliminated when the equalizer is designed to combat various channel conditions with variable equalization tap numbers [1][5]. In a coherent receiving, it is then imperative to extract frequency and phase of the carrier from the incoming signal [6]. Most of the data modems require fast carrier frequency/phase acquisition in the startup and high jitter suppression in the steady state. In practice, it is hard to be satisfied in a simple CR loop, especially when the RF local oscillator (LO) frequency offset has to be taken into account in RF transmission systems [1][3]. In order to reduce the transceiver chip area, the PSF in the transmitter and the LPF in the receiver can be replaced by image rejection lowpass filters (IRLFs) [7]. The IRLF is a linear-phase half-band filter with an V/Q-path interleaving scheme. The hardware cost of the IRLF can be further reduced by eliminating the long delay line which is proportional to the number of coefficient taps.

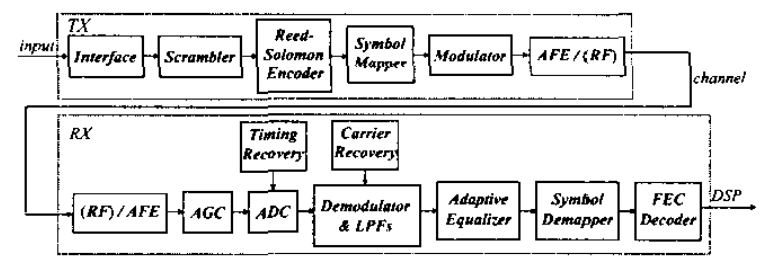

Fig. I. Functional block diagram of QAM transceiver for broadband communications

Based on the QAM transceiver architecture shown in Fig.1, this paper presents a hardware efficient architecture for the multi-stage LMS-based blind equalizer. The proposed pipeline architecture of the equalizer shown in Fig. 3 processes some parallel parts sequentially to reduce the hardware requirement by a factor of four comparing with the architecture implemented directly from the transfer function. Taking the advantage of the $T / 2$ fractionally spaced equalizer, the TR loop shown in Fig. 4 utilizes a baud-rate decision-directed early-late timing extraction scheme to achieve a $\pm 200 p p m$ timing frequency tolerance within $7 \mathrm{~ms}$ acquisition. Since it is mandatory to provide wide locking range and low jitter variation of carrier recovery in a high constellation QAM RF transmission system, the proposed two-fold CR loop can achieve a locking range $\pm 100 \mathrm{kHz}$ (i.e. $\pm 18,500 \mathrm{ppm}$ of the symbol rate) and jitter suppression $-82 \mathrm{dBc}$ for carrier retrieval in a single architecture as shown in Fig.7. In order to further reduce the hardware complexity, the proposed architecture shown in Fig. 2 for the two-phase linear FIR filter can actualize higher hardware efficiency than an IRLF can do.

The organization of this paper is as follows. The transceiver design is presented in Section II. Section III presents VLSI implementation considerations of the transceiver. Section IV shows the simulation results, and the conclusion will be drawn in section $\mathrm{V}$.

\section{TRANSCEIVER ARCHITECTURE}

In this transceiver VLSI architecture design, the 64-QAM $4.035 \mathrm{MHz}$ low-IF digital CATV system is demonstrated. Based on the various digital CATV channel models [8], all the functional blocks are designed to overcome the non-ideal transmission circumstances.

\section{A. Transmitter}

In the proposed QAM transmission system, in order to avoid the drawback of analog demodulation, such as DC offset and mismatch between quadrature paths, the low-IF $4.035 \mathrm{M} \mathrm{Hz}$ modulator is implemented in digital domain. In addition, the anti-sinc FIR filter 
with linear phase is used to compensate the inherent DAC distortion. The square-root raised-cosine filters with a roll-off factor 0.1152 are employed for both transmitter PSFs and receiver LPFs in the CATV system. The proposed two-phase linear FIR filter architecture shown in Fig.2 translates the linear phase FIR filter into a linear phase halfband filter. Using the two-phase clocking technique, the inphase data are latched by the negative clock edge in upper registers while the quadrature data are latched by the positive clock edge in the lower registers. The mutiplexers (MUXs) are controlled by the clock phase to pass the in-phase data to the adders when the clock is high. In contrast, the quadrature data is passed to the adders when the clock is low. Comparing with the traditional quadrature direct form FIR filter, which is composed of $2 N$ multipliers and $(2 N-2)$ adders, the hardware complexity is reduced to $(N+1) / 2$ multipliers and $(N-1)$ adders, where $N$ is the tap number of the quadrature PSF. The power consumption is approximately half of the traditional quadrature direct form FIR filter.

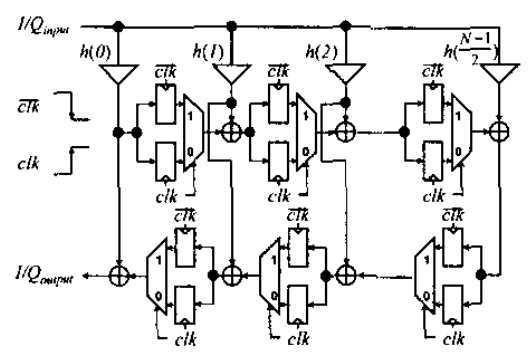

Fig. 2. The proposed two-phase architecture for the pulse shaping filter

\section{B. Adaptive Equalizer}

Based on the multi-stage and signed delay-LMS algorithm [8], the modified LMS algorithm is shown in the following.

$$
C(n+1)=C(n)+\mu \cdot r(n-1) \cdot \operatorname{sgn}\left(e^{*}(n-1)\right)
$$

where $C(n)$ is the adaptive complex coefficient. $\mu$ is the updating step size, which is designed to be a number of power of two for scaling the signal $r(n-1) \cdot \operatorname{sgn}\left(e^{*}(n-1)\right)$ in each adaptive stage with a factor of two to eliminate multiplications. $e(n)$ and $r(n)$ are the error message and the received signal, respectively, and the complex multiplier is replaced by a two's complementer. A stop-and-go flag mechanism [9] associated with $\mu$ is introduced for blind updating. The reduced constellation algorithm $(\mathrm{RCA})$ is adopted to blindly start the equalizer in the acquisition state.

If the direct form and fully parallel process are employed to construct complex filter banks, it will consume $(4 N+4 M)$ real multipliers and adders, where $N$ and $M$ are FF and FB tap numbers respectively. In order to reduce the hardware cost and circuit complexity at the same time, the 8-stage pipeline architecture shown in Fig.3 is proposed. The $c l k$ in Fig.3 is 8 -times of symbol clock rate, i.e. $43.04 \mathrm{MHz}$. The complex-value convolution is sequentially performed by four real value convolutions during the preliminary three hardware stages. After decreasing delays, the real value convolution results are ready at $T 3$ to $T 6$, and complex-value sums, slicing, equalized signal SNR calculations and $1 / 8$ decimation are performed in the final stages, $T 6$ and $T 7$. The proposed pipeline architecture of the blind adaptive equalizer consumes $(N+M)$ real multipliers and adders which is only a quarter of the architecture implemented directly from the transfer function. The programmable numbers $N$ and $M$ can be easily set up according to the channel condition by the hand-shaking protocol during the startup of the transmission.

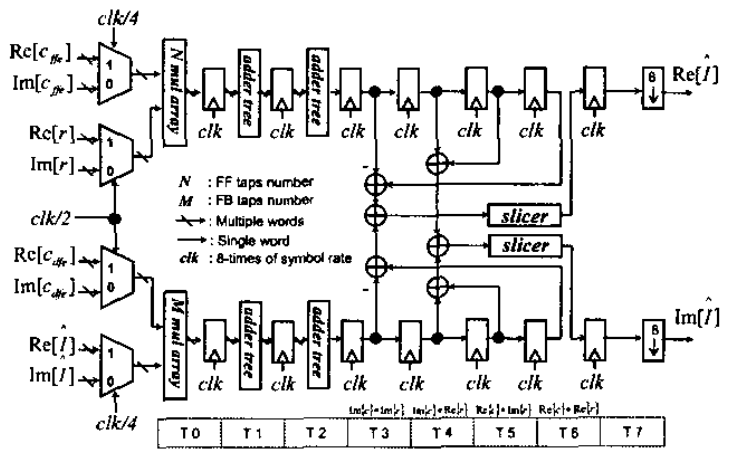

Fig. 3. The proposed 8 -stage pipeline architecture for the adaptive blind equalizer

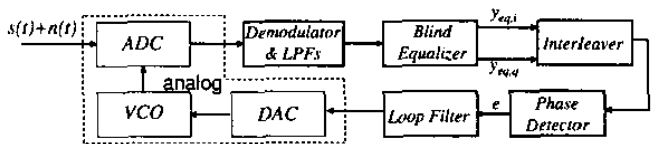

Fig. 4. The receiver architecture using mixed-mode baud-rate timing recovery loop

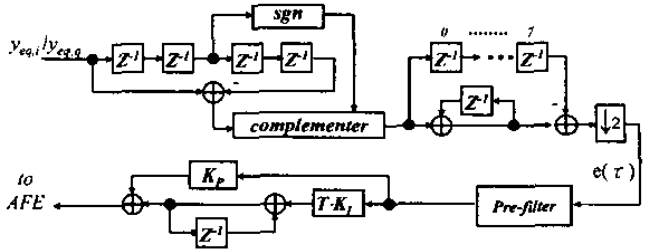

Fig. 5. The phase detector and the loop filter of the timing recovery loop

\section{Timing Recovery}

Instead of suffering signal SNR degradation in an all-digital TR loop with an interpolator, the mixed-mode TR loop architecture shown in Fig. 4 is devised. Practically, the stochastic estimation technique cooperated with a digital phase-locked-loop (PLL) is employed to maintain system stability. Based on the baud-rate early-late timing estimation algorithm shown in Eq.(2) [10], the decision-directed early-late timing estimation derived in Eq.(3) is proposed.

$$
\begin{gathered}
e(\tau)=\operatorname{Re}\left\{A_{n} \cdot\left[y_{e q}(T, \tau)-y_{e q}(-T, \tau)\right]\right\} \\
e(\tau)=E\left\{\operatorname{sgn}\left(\hat{I}_{n}(\tau)\right) \cdot\left[y_{e q, n+1}(\tau)-y_{e q, n-1}(\tau)\right]\right\}
\end{gathered}
$$

where $A_{n}$ and $y_{e q}$ are transmitted and equalized signals, respectively, and $\hat{I}_{n}$ is the decision result. The digital multiplier can be avoided by using the sign bit of the slicer output. Fig.5 shows the circuit of the phase detector and the loop filter of the baud-rate TR loop. Preceded the proportional and integral (PI) loop filter, an IIR prefilter is employed to suppress the noise power. This pre-filter is of 5 to 10 times wider bandwidth than the loop filter, and the closed loop architecture can be treated as a second order PLL. 


\section{Carrier Recovery}

The proposed $\mathrm{CR}$ loop has three operation stages [6]. In the acquisition state, the two-fold CR loop is configured to be the prior CR loop, a modified Costas loop shown in Eq.(4), and starts at time $A$ as shown in Fig.6(a). At time $B$, the two-fold CR loop is switched to the posterior CR loop when the adaptive equalizer converges roughly. Meanwhile, the DC component of the prior $\mathrm{CR}$ loop output, $\omega_{d c}$, is extracted and added to the initial frequency of the numerical-control-oscillator (NCO). The posterior CR loop begins with the NCO frequency at $\omega_{0}+\omega_{d c}$ as shown in Fig.6(b). In this tracking state, the equalizer operates in an ISI-affected converged mode and the CR performs decision-directed maximal-likelihood (DD-ML) phase estimation derived in Eq.(5). At time $C$, the equalizer fully converges and the CR loop is further switched to a decisiondirected minimum-mean-square-error (DD-MMSE) phase estimation shown in Eq.(6). Using the equalized signal, the CR loop provides high jitter suppression in the steady state. The proposed architecture shown in Fig. 7 is hardware efficient since the three-staged operation and the two-fold architecture share most of the circuit functions.

$$
\begin{gathered}
\hat{\phi}_{M L}=-\operatorname{Im}\left\{\operatorname{sgn}\left(\hat{I}^{*}\right) \cdot y_{l}(t)\right\} \\
\hat{\phi}_{M L}=-\operatorname{Im}\left\{\hat{I}^{*} \cdot y_{e q}(t)\right\} \\
\hat{\phi}_{M M S E}=E\left\{\left[y_{e q}(t)-\hat{I}\right]^{*} \cdot \operatorname{sgn}\left(y_{e q}(t)\right)\right\}
\end{gathered}
$$

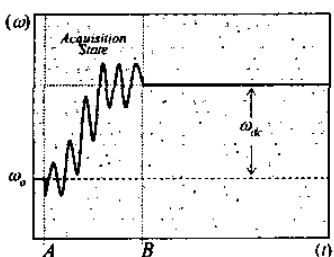

(a)

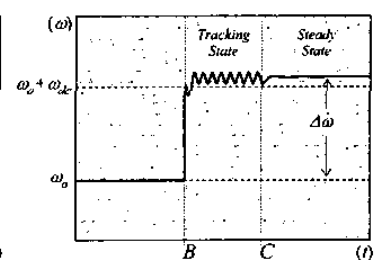

(b)
Fig. 6. The behavior of two-fold carrier recovery loop (a) Prior carrier recovery loop (b) Posterior carrier recovery loop [6]

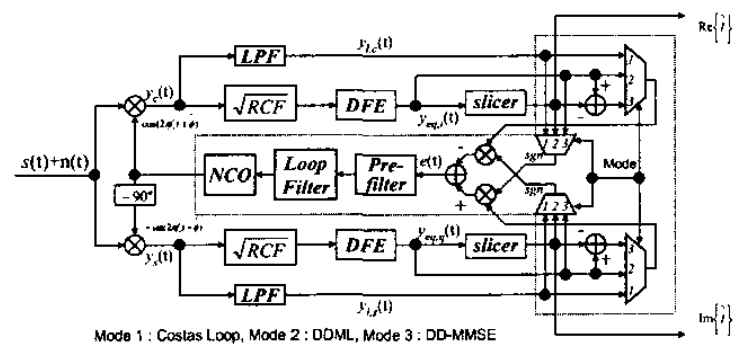

Fig. 7. The proposed architecture to realize the three-staged two-fold carrier recovery loop [6]

\section{VLSI IMPLEMENTATION CONSIDERATIONS}

Three approaches of the VLSI design are employed. The nonregular functional blocks are written in a high level description language (HDL) and synthesized using standard cell methodologies. Then, the clock trees and control signals are manually synthesized to ensure timing constraints. Finally, the dedicated multipliers and adders are synthesized by CAD tools with specifications of delay, area, styles and power. In addition, a self-test circuit is also built in this chip for automatic verification. The head-end transmitter function is also included for system self testing. Using TSMC $0.35 \mu \mathrm{m} 1 \mathrm{P} 4 \mathrm{M}$ CMOS technology, the chip occupies $5.5 \mathrm{~mm} \times 5.5 \mathrm{~mm}$ chip area as shown in Fig.8.

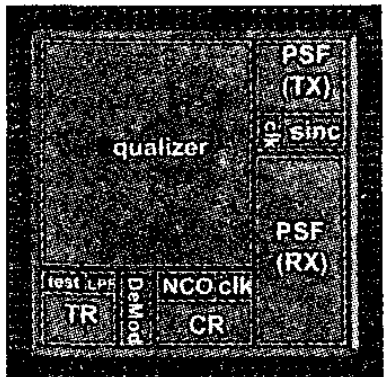

Fig. 8. The layout for $4.035 \mathrm{MHz}$ Low-IF digital baseband of 64-QAM CATV Modem

\section{Simulation Results}

Using the post-layout extracted circuit under 4 process corners, the digital baseband transceiver is simulated in all transmission channel models. The tolerance of the carrier offset in a digital CATV system is $\pm 100 \mathrm{kHz}$, i.e. $\pm 18,500 \mathrm{ppm}$ of the symbol rate, as shown in Fig.9. Fig. 10 shows the frequency convergence of the TR loop when the symbol timing offset is as large as $\pm 200 \mathrm{ppm}$. As soon as the receiver enters the steady state, the carrier jitter can be reduced down to $-82 d B c @ 100 k H z$ away from the carrier frequency, as shown in Fig.11. Fig.12 shows the mean-square-error (MSE) of the equalized signal. The constellation for decision in the steady state is shown in Fig.13. The acquisition time is $7 m s$, which is much less than the requirement of $100 \mathrm{~ms}$ in a CATV system. Table I summarizes the simulation performance of this 64-QAM Low-IF transceiver digital baseband chip.

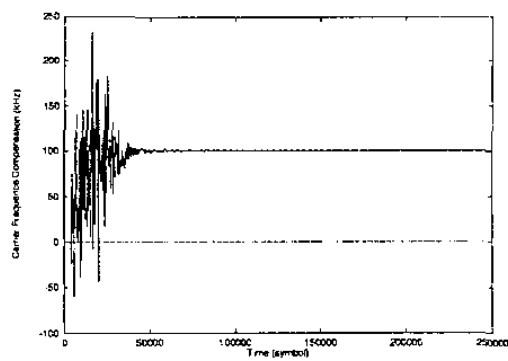

Fig. 9. The $+100 \mathrm{kHz}$ compensation frequency of the carrier recovery loop

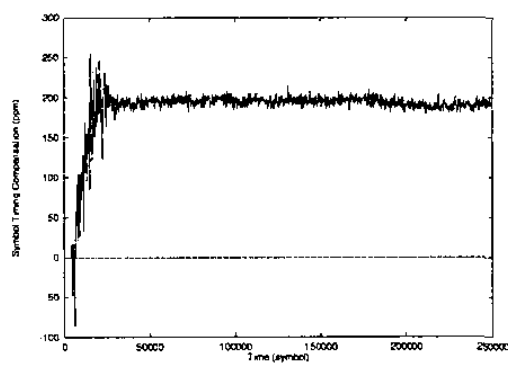

Fig. 10. The $+200 p p m$ compensation frequency of the timing recovery loop 


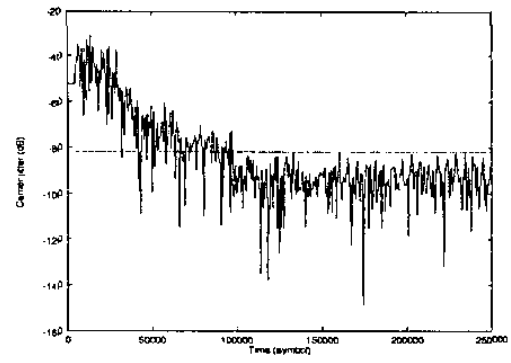

Fig. 11. The carrier jitter performance

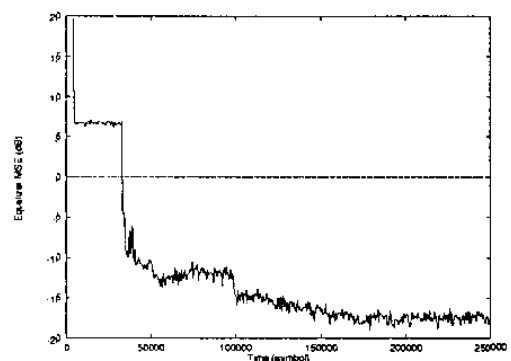

Fig. 12. The MSE of the blind adaptive equalizer

\section{CONCLUSION}

This paper presents a hardware efficient VLSI architecture of digital baseband for 64-QAM low-IF digital CATV system with data rate $32.28 \mathrm{Mb} / \mathrm{s}$. Taking the advantage of the proposed multi-staged LMS based blind equalizer, timing offset tolerance $\pm 200 \mathrm{ppm}$ of the baud rate TR loop and pull-in range better than $\pm 18,500 \mathrm{ppm}$ with $-82 d B c$ jitter suppression of the two-fold CR loop are demonstrated. Both architectures of the adaptive equalizer and the two-phase linear pulse shaping filter contribute the most hardware reduction in this transceiver chip. Since single carrier modulation (SCM) technology is considered one of the main candidates for the Wireless MAN system [3], the proposed architecture, which contains the transmitter, synchronous loops and the programmable blind equalization, can be applied to fixed broadband wireless access networks.

\section{REFERENCES}

[1] Digital Audio-Visual Council, "Lower layer protocols and physical interfaces," DAVIC 1.2 Specification Part 8, Rev. 4.2, 1997.

[2] TIE1.4, "Vdsl technical specification, part 2: Technical specification for a single-carrier modulation (scm) transceiver." Working Group TIEI (DSL Access), Greensboro, NC, February 2001.

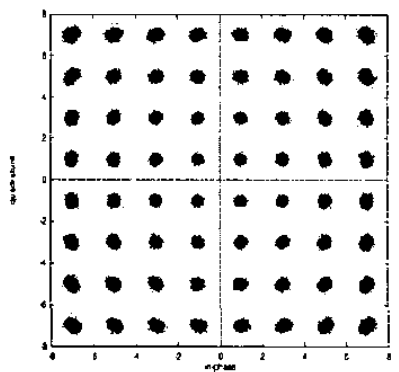

Fig. 13. The signal constellation for decision in the steady statc
TABLE I

THE 64-QAM TRANSCEIVER DIGITAL BASEBAND SIMULATION PERFORMANCE

\begin{tabular}{|c|c|}
\hline Application System & Low.IF digital CATV Modem \\
\hline System Clock & $43.04 \mathrm{M} \mathrm{Hz}$ \\
\hline Symbol Rate & $5.38 \mathrm{M} \mathrm{Hz}$ \\
\hline IF Frequency & $4.035 \mathrm{M} \mathrm{Hz}$ \\
\hline PSF/LPF Tap Number & 39 \\
\hline Anti-sinc Filter Tap Number & 9 \\
\hline Equalizer Tap Number & $\mathrm{FF}: 21, \mathrm{FB}: 19$ \\
\hline Timing Recovery Tolerance & $\pm 200 \mathrm{ppm}$ \\
\hline Carrier Recovery Tolerance & $\pm 100 \mathrm{kHz}$ \\
\hline Carrier Jitter Performance & $-82 \mathrm{dBc} @ 100 \mathrm{kHz}$ \\
\hline Equalizer SNR Performance & $30 \mathrm{~dB}$ \\
\hline Receiver Acquisition Time & $7 \mathrm{~ms}$ \\
\hline Process Technology & TSMC $0.35 \mu \mathrm{m} \mathrm{IP4M}$ \\
\hline Gate Count & 280,195 \\
\hline Supply Voltage & $3.3 \mathrm{~V}$ \\
\hline Power Consumption & $1.35 \mathrm{~W}$ (RX:1.0W) \\
\hline Chip Size & $5.5 \mathrm{~mm} \times 5.5 \mathrm{~mm}$ \\
\hline
\end{tabular}

[3] IEEE Standard for Local and Metropolitan Area Networks, "Part 16: Air interface for fixed broadband wireless access systems-amendment 2: Medium access control modifications and additional physical layer specifications for 2-11 ghz," April 2003.

[4] C. F. Wu, M. T. Shiue, C. C. Huang, and S. J. Jou, "Qam/vsb dual mode equalizer design and implementation," AP-ASIC, pp. $323-326$, Aug 1999.

[5] T1E1.4, "Very-high bit-rate digital subscriber lines (vdsl) metallic interface, part 1: Functional requirements and common specification," Working Group TIEI (DSL Access), Vancouver, Canada, February 2002

[6] C. C. Chang, C. C. Lin, M. T. Shiue, and C. K. Wang, "A wide pull-in range fast acquisition hardware-sharing two-fold carier recovery loop," ISCAS, vol. 4, no. 12, pp. 358-361, May 2001.

[7] L. K. Tan, J. S. Putnam, and F. Lu, "A 70-mb/s variable-rate 1024-qam cable receiver ic with integrated 10-b adc and fec decoder," IEEE $J$. Solid State Circuit, vol. 33, no. 12, pp. 2205-2218, Dec 1998.

[8] M. T. Shiue, Transceiver VLSI Design for High Speed Local Access Modems, Ph.D. thesis, National Central Univ., Taiwan R.O.C., Sept 1998.

[9] G. Picchi and G. Prati, "Blind equalization and carrier recovery using a stop-and-go decesion-directed algorithm," IEEE Trans. Commun., vol. COM-35, no. 9, pp. 877-887, Sept 1987.

[10] K. H. Mueller and M. Muller, "Timing recovery in digital synchronous data receivers," IEEE Trans. Commun., vol. COM-24, pp. 516-531, May 1976. 